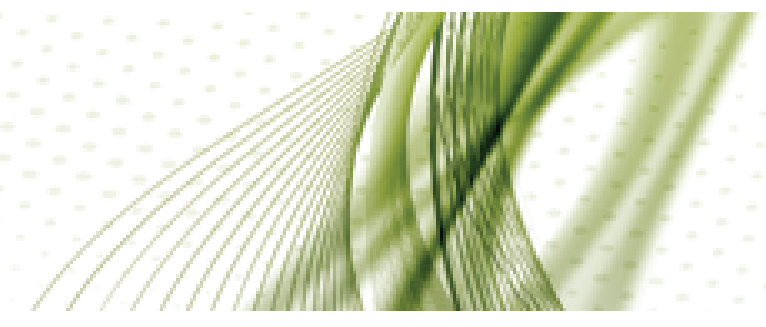

DOI: 10.22374/jmhan.v3i1.33

\title{
SOWING A SEED OF SAFETY: PROVIDING CULTURALLY SAFE CARE IN ACUTE CARE SETTINGS FOR PEOPLE WHO USE DRUGS
}

\author{
Jane McCall, PhD, MSN, RN ${ }^{1}$ and Bernie Pauly, RN, $\mathrm{PhD}^{2}$ \\ ${ }^{1}$ Nurse Educator \\ ${ }^{2}$ Associate Professor in the Faculty of Nursing at the University of Victoria
}

Corresponding Author: janemccall1@gmail.com

\begin{abstract}
This paper reviews the concept of cultural safety from the perspective of people who use illicit drugs and nurses in a hospital setting.

\section{Background}

Illicit drug use is often highly stigmatized and people who use illicit drugs often report negative healthcare experiences contributing to inequities in health and access to healthcare. Registered nurses play a key role in the delivery of healthcare when people who use drugs are hospitalized but often face difficulties in the provision of care. We explored understandings and meanings of cultural safety in healthcare as an approach to mitigate stigma and to promote health equity.

\section{Design and Methods}

Within an overall participatory approach to the research, we employed a qualitative ethnographic approach undertaking 275 hours of participant observation and conducting 34 open-ended interviews with 15 patients and 19 nurses on two acute care hospital units in 2012 and 2013. Result/Findings: Culturally safe care requires recognizing stereotypes and power imbalances; prioritizing trust and building relationships as important outcomes; giving patients space and time; and addressing conflicting organizational values and policies.
\end{abstract}

\section{Conclusions}

Providing culturally safe care requires organizational culture shifts that recognize the importance of historical, societal, and political forces that influence the way in which illicit drug use and people who use illicit drugs are constructed in society.

Keywords: HIV, drug use, safety, health inequity

J Mental Health Addic Nurs Vol 3(1):e1-e7; May 31, 2019.

This article is distributed under the terms of the Creative Commons Attribution-Non

Commercial 4.0 International License. CMcCall and Pauly. 
People who use illicit substances are at risk for harms of drug use including blood-born infections, overdoses, stigma, and violence and experience health inequities or unfair differences in health outcomes as a result of historical, political, social and economic conditions in society. They often die prematurely, have poorer health and experience difficulties in accessing timely medical care. ${ }^{1-3}$ People living with HIV who have substance use issues are at particular risk for such issues as they require ongoing health care and follow up to manage complications and stay well.

HIV and illicit substance use are often highly stigmatized in society affecting health and access to health care. ${ }^{4-6}$ In addition, stigma and living conditions associated with poverty and homelessness also play a role in the development of health inequities for people already at risk for poorer health associated with HIV and substance use.

In his seminal work, Goffman defines stigma as an attribute or characteristic that is considered deeply discrediting by the society or culture to which the individual possessing the attribute belongs. ${ }^{7}$ Stigma has been conceptualized as a process in which differences in power between groups allows one group to name differences and label and stereotype others on the basis of particular behaviors or characteristics. ${ }^{8,9}$ More recently, newer understandings of these differences in power reflect deeply embedded structural and social conditions, which contribute to the enactment of stigma in health care and other types of relationships. ${ }^{10}$

\section{PEOPLE WHO USE ILLICIT DRUGS AND THEIR EXPERIENCES IN HEALTH CARE}

There is a breadth of literature that outlines the issues that people who use illicit drugs face when they attempt to access health care. Nurses experience considerable difficulties in caring for this population. ${ }^{11,12}$ Language often reflects embedded stigma and attention to terms is important in understanding and addressing stigma. For example, using the term "substance abuse" instead of the more neutral term "substance use" is common in the general population and among health care providers. Similarly, health care providers and others often refer to people as "drug users," "addicts," or "illicit drug users" which are highly marginalizing and often dehumanizing terms. Further, nurses' attitudes towards this population have been found to be negative and are often influenced by societal stigma. ${ }^{13}$ This extends to nursing students who also hold stigmatizing attitudes towards patients who use substances. A study by Aggarwal, Ghai and Basu revealed that students saw patients with substance use disorders as being unpleasant, difficult and unworthy of care. ${ }^{14}$ The attitudes of nurses and nursing students appear to be influenced by prevailing attitudes in society at large, which generally sees illicit drug users as dangerous and immoral. ${ }^{15}$

Even more concerning is that nurses hold markedly inaccurate beliefs about pain control and addiction with many assuming that long term use of opioids leads to addiction and that patients with addiction issues who are experiencing pain engage in drug seeking behavior. A study by Krokmyrdal and Andenaes revealed that a majority of nurses surveyed believe that patients with addiction issues exaggerate their pain, are dishonest when describing the effect of their pain medication and misrepresent their pain experience. ${ }^{16}$ These authors and others make apparent that nurses are generally ill prepared to deal with this population. Frequently, nurses receive little in the way of training and education on substance use or care of people who use substances including those experiencing addiction. Drug and alcohol related education comprises a very small proportion of nursing curricula. ${ }^{17}$

Numerous studies have revealed that people who use illicit drugs and are socially disadvantaged are less likely to access primary care, more likely to miss appointments, more likely to use the emergency department, have unmet social services needs and are less likely to take antiretroviral therapy. ${ }^{18}$ Patients are fearful of access-ing care, both as outpatients and inpatients because they fear that they will be subjected to judgmental attitudes from the health care staff. They are made to feel that they are not worthy of receiving help and that they are wasting valuable time. Thus, people who use substances are often reluctant to access health care and may not only avoid but delay accessing health care which highlights the role that stigma plays in the development of health inequities.

J Mental Health Addic Nurs Vol 3(1):e1-e7; May 31, 2019.

This article is distributed under the terms of the Creative Commons Attribution-Non

Commercial 4.0 International License. CMcCall and Pauly. 


\section{PROVIDING A SENSE OF SAFETY}

Clearly, in such situations, people feel unsafe about accessing health care. Cultural safety has been proposed as a response to address concerns about safety in health care especially for indigenous peoples. ${ }^{19}$ Cultural safety was first proposed by Maori nurses working in New Zealand as a way of ensuring that indigenous (Maori) peoples would feel safe accessing care. Maori peoples have historically been reluctant to access the health care system and have disproportionately high rates of mortality and morbidity. ${ }^{20}$

Cultural safety is about recognizing that as a health care worker you come from a position of privilege and power. ${ }^{21,22}$ The goal of cultural safety is to reduce the tendency for health care practices that cause patients to feel unsafe and powerless. It is important for practitioners to understand how their values and beliefs impact on the therapeutic relationships they form with their patients. When practitioners take a culturally safe approach to the care they provide it should help them to resist the temptation to pigeonhole and stereotype based on their beliefs about the group to which they think a patient belongs and to approach each patient as an individual, with their own particular needs and desires that need to be taken into account if a therapeutic relationship based on equity is to be formed. ${ }^{23}$ Some authors have suggested that cultural safety may be relevant to the care of other marginalized populations such as people who use drugs. ${ }^{22}$

\section{CULTURAL SAFETY AND PEOPLE WHO USE DRUGS}

In reflecting on what we know about the experiences of people who use illicit drugs in acute care settings and the applicability of cultural safety, we determined that there was no literature on how cultural safety can be utilized with this population. As a group of academic researchers and practitioners we decided to conduct an ethnographic study examining the experiences of people who use illicit drugs in acute care, their perspectives about what makes them feel safe and unsafe, and the perspectives of front-line nurses providing the care. The research questions for this study were:
1.What constitutes culturally safe care from the perspectives of the patients who use substances and are street involved?

2. What are the strategies that RNs employ to promote the delivery of culturally safe care?

3. What are the similarities and differences in nurse and patient perspectives of culturally safe care?

4. What are the necessary elements of the context that promotes culturally safe care?

5. What are key competencies necessary to provide culturally safe care to patients who use substances and are street involved while they are admitted to hospital?

This study was approved by both the University of Victoria and the hospital ethics review board where the study was conducted. The names of the participants were anonymized. Patient participants were offered a cash honorarium for their time.

The study took place on two acute care medical units in a tertiary care urban hospital. One of the units had a special focus on HIV and urban health. Data collection consisted of 34 qualitative open interviews with both nurses (19) and patients (15). 275 hours of participant observation took place on the same two units and hospital policies related to substance use were reviewed. Eight of the patients were HIV infected. The interviews were transcribed and reviewed for accuracy and $\mathrm{N}$ vivo was used to organize and code the data. Interpretive description was the approach we used to analyse the data. Interpretive description is especially suitable for this type of study because it highlights the constructed and contextual nature of human experience while allowing for shared realities. It is particularly good at turning a lens to everyday nursing practice..$^{24,25}$

The study revealed some interesting findings. ${ }^{26}$ Patients told us they felt judged and labeled as a junkie or an addict. One patient commented: 
No, I think that those people on the [Unit A] floor don't like people that use drugs whether they use drugs now or used drugs a few years ago, they combine it altogether, I think they're very, very, discriminatory about people that, that are addicts. The patient participants felt that they were under surveillance and that the staff was always suspicious of their behaviour.

An item had gone missing and the staff searched the patient's room:

Patient: Well if they had searched once I could see it. After I had told them I had already cleaned up from the search and, no, they're not here. Searching once I can see. Twice to me is just a little suspect. Like, you know, what now you figure that since they were there that I moved them or something and changed the location? 'oh, she's gone, I'll switch locations!'. Because, you know, whatever. It just sounds pretty hokey to me, that's all, you know, it's kind of insulting. Not even kind of, to me it's highly insulting. As I said if I was some old man, they wouldn't have questioned me even once. They would have taken a quick look and went, oh, okay, yeah, it's not here. If you've been involved in drugs, or there's drugs on your record, then anything happens, that's wrong, you're always the first one to be looked at. [pause]. That's a given.
Patients also told us what made them feel safe. In particular, they appreciated it when nurses trusted them and listened to them. They also appreciated it when nurses were attentive to the "small things" such as a glass of juice and a warm blanket.

Patient: They'll take that extra couple of minutes. And you know, see that you're okay. "Let me grab you a blanket" or you know, "Are you sure you don't need something for pain?"You know, you can tell just by their voice that there's genuine concern.

When nurses took time to get to know them and understand their concerns, they felt welcome and less judged. ${ }^{27}$

While patients felt under surveillance, nurses refuted seeing their patients as criminals and saw the criminal justice systems approach to people who use illicit drugs as the real problem. ${ }^{26}$

Nurse: I could talk about it for days. But I don't, I don't, yeah, no, I think drugs should be legal. Not that I'm a drug user or supporter of using drugs but, yeah, I think that drug prohibition does a lot more harm than it does good.

Some of the nurses expressed discomfort with the behavior of patients with problematic substance use whereas others seemed to have a broad perspective on the socioeconomic issues that influenced these patients' lives and ultimately their behavior. This latter perspective embodied a stronger social justice

J Mental Health Addic Nurs Vol 3(1):e1-e7; May 31, 2019.

This article is distributed under the terms of the Creative Commons Attribution-Non

Commercial 4.0 International License. CMcCall and Pauly. 
orientation and an understanding of the factors shaping people's lives.

While the nurses expressed support and integration of a harm reduction approach as part of providing culturally safe care, they expressed discomfort, uncertainty and confusion about the hospital's lack of harm reduction policies. Some had concerns about the substance use policy which required security to be called if substance use was observed. Unfortunately, because of the hospital's anti-drug policy, the nurses did not take the opportunity to talk to their patients about their drug use and ensure that they were using safely and they felt unable to provide clean supplies for those in need of them. ${ }^{26}$ As a result, they often ignored or turned a "blind eye" to substance use in hospital leaving patients at risk and missing opportunities to prevent harm.

\section{DISCUSSION}

It was apparent from our research that patients who use illicit substances including those who are HIV infected felt unsafe accessing health care in the acute care setting. They tended to delay seeking health care until they felt they had no other option and were quick to discharge themselves against medical advice. A number of the patients related stories of feeling marginalized, judged and stigmatized when accessing healthcare.

In our analysis, we found common attitudes towards drug use including judging and blaming people for their substance use without recognizing the conditions that create vulnerability to substance use and criminalizing and/or medicalizing substance use with the stereotyping of people who use drugs as criminals or addicts. ${ }^{26}$ However, the patients also told us stories of times when things had gone well. It was evident that at times they experienced culturally safe care when nurses took time to get to know them, were respectful of their need for space and addressed their needs. When patients received care that was culturally safe, they were happy to stay and were more likely to form therapeutic relationships with the staff.

\section{DISSEMINATION AND IMPACT}

We presented our research findings at two forums that were attended by front line nurses and administrators as well as people from two drug user groups at two hospitals, including the study hospital. These forums provided an opportunity for presentation of the research and then a space for people who use drugs and nurses who care for them to enter into a dialogue about substance use, stigma and the meaning of culturally safe care. It gave nurses the opportunity to reflect on the societal and systematic attitudes towards substance use and stigma experienced by people who use drugs. Together, the attendees reflected on the research findings and contributed to the development of culturally safe guidelines for people who use drugs and are admitted to acute care. Following the forum, these recommendations were further refined with the assistance of a local drug user group, the Society of Living Illicit Drug Users and a research bulletin was created. ${ }^{27}$

Among the recommendations that we developed were that culturally safe care needs to foster the engagement and participation of people who have experience of substance use and marginalization in the delivery of care. Care givers need to recognize that people's health, health care, priorities and experiences are influenced by history and policies that criminalize drug use. It is important for health care staff to consider how past histories of trauma and violence, layers of disadvantage, and stigma may affect a patient's ability to engage with providers and care plans. Trust is not a given and trust must be established as a priority outcome. To achieve a positive relationship and build trust health care providers need to develop a culture of safety and respect where all patients are valued and seen as deserving of care.

As a direct result of our research, the hospital where the study took place has reformed its substance use policy and philosophy of care for people who use substances. The philosophy of care now acknowledges that abstinence is a goal but not a given and that nurses may provide clean injection equipment and crack pipes to their patients. This is a significant change in practice that should go a long way to addressing the needs of this population of patients and contributing to better health care and health care relationships. In the other

J Mental Health Addic Nurs Vol 3(1):e1-e7; May 31, 2019.

This article is distributed under the terms of the Creative Commons Attribution-Non

Commercial 4.0 International License. CMcCall and Pauly. 
hospital where a forum was held, the cultural safety program mandate was expanded to include patients who are homeless and use illicit drugs.

\section{CONCLUSION}

Cultural safety is especially relevant in the provision of nursing care for people who use illicit drugs and live with social disadvantages such as HIV infection, homelessness and poverty. Cultural safety provides a potential lens for examining the discourses, structures and assumptions that shape the interactions we have with our patients. It is important for nurses to critically reflect on how substance use is taken up and framed in health care and how that impacts on the interactions they have with people who use illicit drugs as part of a culturally safe approach to nursing care. It is also crucial for nurses to consider how they can make acute care safe for their drug using patients. As one nurse in the study put it, nurses need to ". . . plant a seed of safety .... ${ }^{27}$ Specific attention to the elements of culturally safe care that are outlined in this paper holds promise for improving nursing practice in ways that can foster equity and social justice.

\section{REFERENCES}

1. McCoy C, Metsch L, Chitwood D, and Miles C. Drug use and barriers to use of health care services. Subst Use Misuse 2001;36(6\&7):780-806.

2. McNeil R, Small W, Wood E, and Kerr T. Hospitals as a "risk environment": An ethno-epidemiological study of voluntary and involuntary discharge from hospital against medical advice among people who inject drugs. Soc Sci Med 2014;105:59-66.doi: 10.1016/j .socscimed.2014.01.010.

3. Merrill J, Rhodes L, Deyo R, et al. Mutual distrust in the medical care of drug users. J Gen Int Med 2002;17(5):327-33.

4. Lloyd C. Sinning and Sinned Against: The Stigmatization of Problem Drug Users. London, UK: Drug Policy Commission; 2010.

5. Room R. Stigma, social inequality and alcohol and drug use. Drug Alc Rev 2005;24(2):143-55.doi: 10.1080/09595230500102434.

6. Neuman M, Obermyer CM, Cherutich P, et al. Experiences of stigma, discrimination, care and support among people living with HIV: A four country study. AIDS Behav 2013;17(5):1-21. doi: 10.1007/s10461-013-0432-1.
7. Goffman E. Stigma: Notes on the Management of a Spoiled Identity. Englewood Cliffs: Prentice Hall; 1963.

8. Link B and Phelan J. Conceptualizing stigma. Ann Rev Sociol 2001;27:363-85.

9. Link B and Phelan J. Stigma and its public health implications. The Lancet 2005;367(9509):528-29. doi: 10.1016/50140-6736(06)68184-1.

10. Link BG and Phelan J. Stigma power. Soc Sci Med 2014;103:24-32. doi: 10.1016/j.socscimed.2013.07.035.

11. Browne AJ, Smye VL, Rodney P, et al. Access to primary care from the perspective of Aboriginal patients in an urban emergency department. Qualitative Health Research 2011;21(3):33-348. doi:10.1177/1049732310385824.

12. van Boekel LC, Brouers EP, van Weeghel J, and Garretsen HF. Stigma among health professionals towards patients with substance use disorders and its consequences for health care delivery: A systematic review. Drug Alc Depend 2013;31(1):23-35.

13. Natan MB, Beyil V, and Neta O. Nurses' perception of the quality of care they provide to hospitalized drug addicts: Testing the Theory of Reasoned Action. Int J Nursing Pract 2009;15(6):566-73. doi: 10.1111/j.1440-172X.2009.01799.x.

14. Aggarwal M, Ghai S, and Basu D. Attitude of nursing students towards psychoactive substance use: Does training matter? Int J Nurs Educ 2012;4(2):146-50.

15. Strike GJ, Myers T, and Millson M. Finding a place for needle exchange programs. Crit Pub Health 2004;14(3):261-75. doi: 10.1080/09581590400004386.

16. Krokmyrdal KA and Andenaes R. Nurses' competence in pain management in patients with opioid addiction: A cross-sectional survey study. Nurse Ed Today 2015;35(6):789-94.doi: 10.1016/j. nedt.2015.02.022.

17. Campbell-Heider N, Finnell DS, Feigenbaum JC, et al. Survey on addictions: Towards curricular change for family nurse practitioners. Int J Nurs Educ Scholarship 2009;6(1):1-17.doi: 10.2202/1548-923x.1645.

18. Sohler NL, Wong MD, Cunningham WE, et al. Type and pattern of illicit drug use and access to health care services for HIV infected people. AIDS Patient Care STDs 2007;21(S1):S68-S76. doi: 10.1089/ apc.2007.9985.

19. Papps E and Ramsden I. Cultural safety in nursing: The New Zealand experience. Int J Qual Health Care 1996;8(5):491-97.

20. Ramsden J. The Treaty of Waitangi and Cultural Safety: The Role of the Treat in Nursing and Midwifery

J Mental Health Addic Nurs Vol 3(1):e1-e7; May 31, 2019.

This article is distributed under the terms of the Creative Commons Attribution-Non

Commercial 4.0 International License. @McCall and Pauly. 
Education in Aortearoo. Wellington: Nursing Midwifery Council; 1996.

21. Anderson J, Perry J, Blue C, et al. "Rewriting" cultural safety within the postcolonial and postnational feminist project: Toward new epistemologies of healing. Advances in Nursing Science 2003;26(3):196-214.

22. Browne AJ, Varcoe C, Smye V, et al. Cultural safety and the challenges of translating critically oriented knowledge in practice. Nursing Philosophy 2009;10(3):167-79. doi: 10.1111/j.1466-769X.2009.00405.x.

23. McCall J and Pauly B. Providing a safe place: Adopting a cultural safety perspective in the care of Aboriginal women living with HIV/AIDS. Can J Nurs Res 2012;44(2):130-45.
24. Thorne S. Interpretive Description. Walnut Creek, CA: Left Coast Press; 2008.

25. Thorne S, Reimer Kirkham S, and O'Flynn-Magee. The analytic challenge in interpretive description. Int J Qualitatat Meth 2004;3(1):1-21.

26. Pauly B, McCall J, Browne AJ, et al. Toward cultural safety: Nurse and patient perceptions of illicit substance use in a hospitalized setting. Adv Nurs Sci 2015;38(2):121-35. doi: 10.1097/ANS.0000000000000070.

27. Pauly B, McCall J, Parker J, et al. Creating Culturally Safe Care in Hospital Settings for People Who Use(D) Illicit Drugs, Bulletin \#11. Victoria, BC: University of Victoria, Centre for Addictions Research of BC; 2013. 